\title{
Editorial
}

Brendan Finucane

\section{The difficult airway - a Canadian perspective}

"If at first you don't succeed, don't take any more chances"

Frank "Kin" Hubbard

$1868-1930$

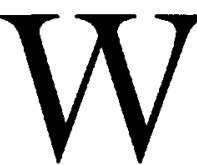

ILLIAM Macewen ${ }^{1}$ first performed tracheal intubation 120 years ago in a patient who had a large tumour in his oropharnx. Several more years would elapse before this technique became a routine procedure during general anaesthesia. Although a large number of individuals contributed to the evolution of this technique, two very important events sparked the routine use of endotracheal anaesthesia: the introduction of the Macintosh laryngoscope and the neuromuscular blocking drugs. Although barely acknowledged by our colleagues in other specialities, endotracheal anaesthesia has paved the way for some of the greatest advances in modern surgery. Tunstall ${ }^{2}$ was the first to articulate that we should develop a more organized approach to airway management when he introduced the concept of the "difficult airway drill", when dealing with airway difficulties in obstetric patients. The first guidelines on the management of the difficult airway were published by the ASA in 1993. ${ }^{3}$ These guidelines have been updated once since then, following the introduction of the Laryngeal Mask Airway in the US. ${ }^{4}$ There have been more publications on airway management issues in the last decade, than in the previous 50 years. A review of Cumulative Index Medicus from 1930 to 1939 , indicated that there were 68 publications on airway management issues. A similar review from 1984 to 1994 showed that there were 400 such publications. ${ }^{5}$

In this edition of the Journal, Crosby et al. (The Canadian Airway Focus Group $)^{6}$ have written an excellent article best described as a "review with recommendations" on the topic of the unanticipated difficult airway. This review serves as a "state of the art" treatise on the unanticipated airway. It is very concise, readable and inclusive and displays a distinctively Canadian view- point, and is an excellent update on this rapidly changing area of anaesthesia and airway management. The algorithm provided is a much simplified version of that presented in the ASA guidelines and could be quickly referred to, even in an emergency setting.

The CAFG definition of the difficult airway is more practical and inclusive than that presented in the ASA guidelines. The ASA Guidelines defined difficult intubation as follows: "requires more than three attempts" or "more than ten minutes." These definitions are liberal by Canadian standards and may reflect the use of non-MD anaesthesia providers in a large number of cases in the US. Ideally, we need universal agreement on these definitions so that we can compare data from different countries. The CAFG reaffirms the importance of airway evaluation in the prevention of airway disasters and emphatically states that no one test will infallibly reveal a difficult airway. Their recommendations strongly concur with the ASA guidelines on this issue. Despite these strong recommendations from both groups, compliance is poor among anaesthetists. Rose $e t a l^{7}$ recently completed a study on airway evaluation in 18,500 patients and noted inadequate data in more than $50 \%$ of cases.

The CAFG correctly state that the only reliable ways to confirm tracheal intubation are by visual inspection (either directly or endoscopically) and by end-tidal $\mathrm{CO}_{2}$ monitoring and point out that clinical signs cannot be relied upon to confirm tracheal placement. Graphic evidence in support of these recommendations can be found in the Closed Claims Study, which reports 188 deaths or serious injuries from oesophageal intubation in the US., since $1975 .^{8}$ This does not mean that we should dispose of our stethoscopes in the operating room, although many already have. Auscultation is still a very useful way of ruling out bronchial intubation.

Two of the most important developments in airway management since tracheal intubation became a routine part of anaesthesia, were fibreoptic assisted intu- 
bation (FAI) and the introduction of the LMA. The CAFG emphasized the importance of FAI in awake patients, but were less enthusiastic about it in anaesthetised patients. I tend to differ with them on this issue. Fibreoptic assisted intubation can be extremely useful even in anaesthetised patients, but like other airway adjuncts, it requires practice, experience and an assistant to maintain the airway, whilst the operator dedicates attention to the task at hand. Specially designed masks are available to facilitate FAI in anaesthetised patients. Fibreoptic assisted nasal intubation can also be performed in anaesthetised patients with the help of an assistant.

The introduction of the LMA has revolutionized the practice of anaesthesia world-wide and is one of the most important advances in both anaesthesia and airway management. ${ }^{9}$ The introduction of the LMA precludes the need for paralysis and tracheal intubation in a huge number of patients world-wide, thereby reducing the risk of morbidity and mortality related to difficult intubation, oesophageal intubation and inadequate ventilation. We need more than one publication to convince us that stimulation generated by LMA insertion is the equivalent to that of tracheal intubation and that the LMA is more likely to promote gastric regurgitation than bag/mask ventilation. The LMA plays a major role not only in prevention, but also in the management of airway problems and is now an integral part of most difficult airway algorithms. I take issue with the CAFG about their recommendations for the "Difficult Airway Kit". They suggest that it should include "an alternative to the facemask" for difficult or failed intubation. We need to be more specific. Perhaps the literature marginally supports the superiority of the Combitube in these circumstances but most anaesthetists are familiar with LMA insertion and not with the Combitube. The LMA has one major advantage over the Combitube, in addition to relieving airway obstruction in a number of cases, it also acts as a conduit for subsequent tracheal intubation.

The CAFG discusses the virtues and drawbacks of other airway adjuncts and emphasize the importance of familiarity with equipment before use in life threatening situations. They also reassure us that we do not need to be masters of all of these intriguing devices. However, they stress the importance of learning how to perform transtracheal ventilation (cricothyrotomy and retrograde technique) because it may be the only option when conventional methods fail. Transtracheal Jet Ventilation (TTJV) is barely mentioned by the
CAFG and the only reference to it, is in the form of a warning. This is probably appropriate until we have more data supporting its use. TTJV should be reserved for desperate situations, when no other options are available. We differ from our confreres in the US on this issue.

One of the most important recommendations made by the CAFG is that relating to formal training in difficult airway techniques. Anaesthetists seem to be more cognizant of the dangers associated with difficult intubation, yet are still reluctant to adopt a protocol approach to this medical emergency. The main reason that we do not handle this emergency well, is that few of us have been trained to do so. A recent survey by Koppel et al. ${ }^{10}$ revealed that only $27 \%$ of 143 anaesthesia programmes in the US participated in a rotation dedicated to the management of the difficult airway and most of these educational endeavours, consisted of lectures only. It seems ironic that we have neglected to advance our skills in this core subject, yet we go to great lengths to acquire eclectic skills in other subspecialty areas. The solution to this problem is obvious. Management of the difficult airway must be an integral part of the curriculum in all anaesthesia residency training programmes. We, in Canada, should take the initiative and play a leadership role in this endeavour. Duncan et al.,"11 estimated that a resident would need to be exposed to 29 separate difficult intubation scenarios, to become proficient. Few programmes in North America can afford this kind of exposure. Therefore, we must establish laboratories that allow us to simulate difficult airway scenarios and allow trainees exposure to new equipment and complicated interventions. There are numerous opportunities to acquire other airway skills during anaesthesia training. One can learn how to use the Bullard laryngoscope, the lighted stylet and other devices in the course of providing routine anaesthesia care during training, yet few trainees seem to avail themselves of this opportunity.

We still have a tendency to rely on our surgical colleagues to rescue us when conventional airway techniques fail. We should not perform procedures on patients unless we are capable of dealing with the complications ourselves. Surgeons may be even less familiar with the equipment required to deal with transtracheal approaches than we are and tend to favour tracheostomy under these circumstances. Unfortunately, few surgeons are capable of performing a formal tracheostomy in less than five minutes. Therefore, the formal tracheostomy has no role in emergency airway management. 
Airway management has changed dramatically in the past two decades. We have seen major advances in airway equipment and monitoring. Operative obstetrics is now predominantly performed under regional anaesthesia in contrast to 30 years ago. We have seen a considerable attitude change among anaesthetists, most of whom now view unanticipated difficult intubation as a serious matter. Have these changes had any impact on outcome? The Closed Claims Study in the US, has been accumulating data on anaesthesia related complications since 1975 . For the first time in three decades, they have reported a decrease in the number of airway related complications. ${ }^{12}$ Whilst it may be difficult to pinpoint the reasons for this change, it is gratifying to report this positive result as we prepare to enter the new millennium.

What have we learned from this review? First of all, the vast majority of difficult airways can be anticipated by performing a thorough evaluation of the airway. Second, if you encounter a difficult airway, make sure that the incident is appropriately documented and that that information is relayed to the physician responsible for that patient's long term care. Arrangements for a Medic-Alert bracelet is warranted in some difficult airway cases. Third, difficult airway training should be a mandatory part of all anaesthesia training programmes. Finally, and perhaps most importantly, each airway intervention must be approached with a contingency plan in mind.

In summary, the CAFG deserves to be congratulated on providing us with an excellent, concise, inclusive treatise on the management of the unanticipated difficult airway. This document is easy to read and is an excellent reference on this rapidly changing area of anaesthesia. The algorithm provided is easy to follow and should be taped on the wall of every operating room suite.

The quotation above by "Kin" Hubbard, the wellknown writer/humourist, was not composed with medical emergencies in mind. However, these words of wisdom seem most fitting in the setting of an unanticipated difficult airway.

\section{Problème d'intubation - un point de vue canadien}

"Si le premier essai ne réussit pas, ne courez pas d'autres risques"

Frank "Kin" Hubbard

$1868-1930$

C'est William Macewen ${ }^{1}$ qui, le premier, a réalisé une intubation endotrachéale il y a 120 ans chez un patient qui avait une grosse tumeur à l'oropharynx. Quelques années encore se sont écoulées avant que cette technique ne devienne une pratique courante pendant l'anesthésie générale. Quoique de nombreux individus aient participé à l'évolution de cette technique, deux événements très importants ont transformé la manière habituelle d'utiliser l'anesthésie endotrachéale : l'arrivée du laryngoscope Macintosh et les myorelaxants. Bien qu'elle soit à peine connue de nos collègues d'autres spécialités, l'anesthésie endotrachéale a favorisé certains des plus grands progrès de la chirurgie moderne. Tunstall ${ }^{2}$ a été le premier à dire que nous devrions élaborer une façon plus méthodique de procéder à l'intubation quand il a présenté l'idée d' "exercices pour l'intubation difficile", devant les difficultés d'intubation auxquelles il a dû faire face avec les patientes en obstétrique. Les premières directives concernant la façon de traiter l'intubation difficile ont été publiées par la ASA en $1993 .{ }^{3}$ Ces directives ont été mises à jour une fois depuis, à la suite de l'introduction du masque laryngé (ML) aux États-Unis. ${ }^{4}$ Il y a eu plus de publications sur des questions se rapportant à l'intubation pendant la dernière décennie que pendant les $\mathbf{5 0}$ années précédentes. Une revue de l'Index Medicus cumulatif de 1930 à 1939 indique 68 publications sur des façons de procéder à l'intubation. Une revue semblable de 1984 à 1994 fait état de 400 publications sur le sujet. ${ }^{5}$

Dans le présent numéro du Journal, Crosby et coll. (Le groupe d'étude canadien sur l'intubation, GÉCI) ${ }^{6}$ a produit un excellent article, mieux défini comme une "revue et recommandations", au sujet des difficultés d'intubation imprévues. Cette revue se présente comme le traité "de pointe" sur l'intubation imprévue. Elle est très concise, facile à lire et complète; de plus, elle expose un point de vue strictement canadien et elle est une excellente mise à jour des connaissances qui concernent l'anesthésie et la démarche d'intubation, des domaines en constante évolution. La marche à suivre proposée est une version plus simple de celle qui était présentée dans les recommandations de l'ASA et on peut s'y référer rapidement, même en situation d'urgence.

La définition de l'intubation difficile par le GÉCI est plus pratique et complète que celle qui est présentée dans 
les directives de l'ASA. Les directives de l'ASA ont donné de l'intubation difficile les caractéristiques suivantes : "nécessite plus de trois essais" ou "plus de dix minutes". Ces définitions manquent de rigueur selon les normes canadiennes et tiennent peut-être compte du fait que l'anesthésie est fournie par des non-médecins dans un grand nombre de cas aux États-Unis. Nous avons besoin, idéalement, d'un accord international sur ces définitions de façon à pouvoir comparer des données en provenance de différents pays. Le GÉCI réaffirme l'importance de l'évaluation de l'intubation pour prévenir les échecs désastreux et il insiste pour dire qu'il n'y a pas de test infaillible qui puisse révéler une intubation difficile. Leurs recommandations appuient fermement les directives de l'ASA à ce sujet. Malgré cette attitude des deux groupes, l'adhésion est faible parmi les anesthésistes. Rose et coll. ${ }^{7}$ ont terminé récemment une étude sur l'évaluation de l'intubation chez 18500 patients et ils ont noté des données erronées dans plus de $50 \%$ des cas.

Le GÉCI affirme précisément que les seules façons fiables de confirmer l'intubation endotrachéale sont l'inspection visuelle (soit directement, soit avec l'endoscope) et le monitorage du $\mathrm{CO}_{2}$ de fin d'expiration ; il fait aussi remarquer qu'on ne peut se fier aux signes cliniques pour confirmer la position endotrachéale du tube. On peut trouver la représentation graphique qui accompagne ces recommandations dans la "Closed Claims Study" qui rapporte 188 morts ou blessures graves provoquées par l'intubation œsophagienne aux Etats-Unis, depuis $1975 .^{8}$ Cela ne veut pas dire que nous devrions nous débarrasser de nos stéthoscopes dans la salle d'opération, quoique nombreux soient ceux qui le font déjà. L'auscultation demeure une manière très utile d'exclure l'intubation bronchique.

Deux des événements les plus importants à survenir depuis que l'intubation endotrachéale fait partie de la pratique courante en anesthésie sont l'intubation à l'aide d'un fibroscope (IAF) et l'arrivée du ML. Le GÉCI souligne l'importance de l'LAF chez les patients éveillés, mais il est moins enthousiaste quand il s'agit de patients sous anesthésie. J'ai tendance à différer sur ce point. L'intubation à l'aide du fibroscope peut être très utile même chez les patients sous anesthésie mais, comme dans le cas d'autres auxiliaires, elle nécessite un entraînement, de l'expérience et un assistant qui maintient la ventilation pendant que l'opérateur se concentre sur sa tâche. Des masques de conception particulière sont offerts pour faciliter l'IAF chez des patients sous anesthésie. L'intubation nasale à l'aide du fibroscope peut être pratiquée aussi chez des patients curarisés en présence d'un assistant.

L'introduction du ML a révolutionné la pratique de l'anesthésie à travers le monde et constitue l'un des plus importants progrès en anesthésie et dans la démarche de l'intubation. ${ }^{9}$ L'arrivée du ML exclut la nécessité de la curarisation et de l'intubation endotrachéale chez un grand nombre de patients dans le monde, réduisant ainsi le risque de morbidité et de mortalité relié à l'intubation difficile, à l'intubation œsophagienne et à la ventilation insuffisante. Il faudra plus d'une publication pour nous convaincre que la stimulation provoquée par l'insertion du $\mathrm{ML}$ est équivalente à celle de l'intubation endotrachéale et que le ML est plus susceptible de produire de la régurgitation gastrique que la ventilation manuelle au masque et au ballon. Le ML joue un rôle majeur non seulement dans la prévention mais aussi dans le traitement des problèmes d'intubation et c'est maintenant une partie intégrante de la plupart des directives concernant l'intubation difficile. Je ne suis pas d'accord avec les recommandations du GÉCI quant au "Matériel pour intubation difficile". Il suggère d'inclure "une alternative au masque" en cas d'échec ou de difficultés d'intubation. Nous devons être plus précis. Peut-être dans la littérature insiste-t-on sur la supériorité du Combitube dans ces circonstances, mais l'insertion du ML est mieux connue de la plupart des anesthésistes, contrairement au Combitube. Le ML présente un grand avantage par rapport au Combitube, en plus de soulager l'obstruction de l'intubation dans de nombreux cas, il sert aussi de conduit pour une intubation endotrachéale subséquente.

Le GÉCI analyse les vertus et les limites d'autres accessoires d'intubation et il souligne l'importance de se familiariser avec l'équipement avant de l'utiliser dans des situations où la vie est en jeu. Il confirme aussi que nous n'avons pas besoin de maitriser tous ces appareils intrigants. Cependant, il insiste sur l'importance d'apprendre comment procéder à la ventilation transtrachéale (cricothyrotomie et technique rétrograde) parce qu'elle peut être la seule solution quand les méthodes classiques ne fonctionnent pas. La ventilation transtrachéale avec jet (VTTJ) est rarement mentionnées par le GÉCI et la seule référence qu'on y fait prend la forme d'une mise en garde. C'est sans doute opportun jusqu'à ce que nous ayons plus de données qui encouragent son usage. La VTTJ devrait être réservée aux situations désespérées, alors que nous ne disposons d'aucun autre choix. Nous nous distinguons de nos confrères des États-Unis sur cette question.

L'une des recommandations les plus importantes du GÉCI concerne l'entrainement formel aux techniques d'intubation. Les anesthésistes semblent plus conscients des dangers associés aux difficultés d'intubation, bien qu'encore réticents à adopter une démarche méthodique dans les situations médicales d'urgence. La principale raison pour laquelle nous ne pouvons bien nous occu- 
per de cette urgence c'est que peu d'entre nous ont reçu une formation pour le faire. Une récente enquête réalisée par Koppelet coll. ${ }^{10}$ révèle que $27 \%$ seulement de 143 programmes d'anesthésie aux États-Unis offrent de participer à tour de rôle à une formation consacrée à la prise en charge de l'intubation difficile, mais la plupart de ces séances d'éducation sont faites de lectures uniquement. Il semble ironique que nous ayons négligé de parfaire nos connaissances de ce sujet fondamental, alors que nous nous donnons beaucoup de mal pour acquérir diverses techniques relevant d'autres sous-spécialités. La solution de ce problème est évidente. La gestion de l'intubation difficile doit être une partie intégrante du contenu de tout programme de formation de la résidence en anesthésie. Nous, du Canada, devrions prendre l'initiative et jouer un rôle de direction dans cette entreprise. Duncan et coll., ${ }^{11}$ croient qu'un résident peut avoir besoin d'être exposé à 29 différentes situations d'intubation difficile pour en acquérir la maîtrise. Peu de programmes en Amérique du Nord peuvent se permettre un entrainement de ce genre. Nous devons donc mettre sur pied des laboratoires où simuler des situations d'intubation difficile et permettre aux stagiaires d'expérimenter du nouveau matériel et des interventions compliquées. Il y a beaucoup d'occasions de développer d'autres habiletés d'intubation pendant la formation en anesthésie. On peut apprendre à utiliser le laryngoscope Bullard, le stylet lumineux et d'autres appareils au cours de la pratique courante de la résidence en anesthésie, néanmoins peu de stagiaires semblent profiter de l'occasion.

Nous avons encore tendance à compter sur nos collègues chirurgiens pour qu'ils viennent à notre secours quand les techniques habituelles d'intubation échouent. Nous ne devrions pas utiliser les techniques avec les patients si nous ne pouvons faire face nous-mêmes aux complications. Il se peut que le matériel requis soit encore moins familier aux chirurgiens qu'il ne l'est pour nous et qu'ils ne puissent se servir des techniques transtrachéales, tendant plutôt à favoriser une trachéotomie dans ces circonstances. Malheureusement, peu de chirurgiens peuvent réaliser une trachéotomie formelle en moins de cinq minutes. Par conséquent, la trachéotomie formelle n'a pas sa place lors d'une intubation urgente.

La gestion de l'intubation a beaucoup évolué depuis vingt ans. Nous avons connu les progrès importants du matériel d'intubation et du monitorage. La chirurgie obstétrique est maintenant réalisée le plus souvent sous anesthésie locorégionale par rapport à ce qui se passait il y a 30 ans. Nous avons vu un grand changement d'attitude parmi les anesthésistes, la plupart d'entre eux s'inquiètent sérieusement maintenant de la possibilité d'une intubation difficile. Ces changements ont-ils eu des conséquences dans la pratique ? La "Closed Clains Study" aux États-Unis a recueilli des données sur des complications reliées à l'anesthésie depuis 1975. Pour la première fois en trente ans, ils ont rapporté une baisse du nombre de complications reliées à une intubation. ${ }^{12}$ Même s'il peut être difficile de préciser les raisons de ce changement, il est satisfaisant de rapporter ce résultat positif au moment où nous nous préparons à entrer dans un nouveau millénaire.

Que nous a appris cette revue ? D'abord, la grande majorité des intubations difficiles peut être prévue en faisant une évaluation approfondie des voies aériennes. Deuxièmement, si vous avez été victime d'un problème d'intubation, assurez-vous que l'incident est suffisamment documenté et que cette information soit transmise au médecin responsable à long terme des soins de ce patient. Dans certains cas d'intubation difficile, il peut être justifié de fournir un bracelet Medic-Alert. Troisièmement, un entraînement aux mesures à prendre en cas d'intubation difficile devrait être obligatoire pour tout programme de formation en anesthésie. Enfin, et c'est peut-être le plus important, chaque intervention sur les voies aériennes devrait être abordée en ayant à l'esprit un plan d'urgence.

En résumé, le GÉCI a droit à notre reconnaissance pour nous avoir fourni un excellent traité, concis et complet sur la façon de procéder lors d'une intubation difficile imprévue. Ce document est facile à lire et constitue une excellente référence dans ce champ de l'anesthésie en évolution constante. La méthode fournie est facile à suivre et devrait être affichée au mur de toute salle d'opération.

La citation du début de "Kin" Hubbard, l'écrivain humoriste bien connu n'a pas été écrite en ayant à l'esprit les urgences médicales. Toutefois, ces mots de sagesse semblent convenir exactement à la marche à suivre en cas d'intubation difficile non anticipée.

\section{References}

1 James $C D T$. Sir William Macewen and anaesthesia. Anaesthesia 1974; 29: 743-53.

2 Tunstall ME. Failed intubation drill. Anaesthesia 1976; 31: 850.

3 Caplan RA, Benumof JL, Berrry FA, et al. Practice guidelines for management of the difficult airway. A report by the American Sociery of Anesthesiologists Task Force on Management of the Difficult Airway. Anesthesiology 1993; 78: 597-602.

4 Benumof JL. Laryngeal mask airway and the ASA difficult airway algorithm. Anesthesiology 1996; 84: 686-99.

5 Finucane BT, Santona AH. Principles of Airway Management. St. Louis: Mosby 1996 (Foreword viii). 
6 Crosby ET, Cooper RM,Douglas MJ, et al. The unanticipated difficult airway with recommendations for management. Can J Anaesth 1998; 45: 757-76.

7 Rose $D K$, Coben $M M$. The airway: problems and predictions in 18,500 patients. Can J Anaesth 1994; 41 : 372-83.

8 Cheney FW. ASA refresher course lectures, 1996.

9 Brain AIJ. The laryngeal mask - a new concept in airway management. Br J Anaesth 1983; 55: 801-5.

10 Koppel JN, Reed AP. Formal instruction in difficult airway management. A survey of anesthsiology residency programs. Anesthesiology 1995; 83: 1343-6.

11 Duncan $P G$, Cohen $M M$, rip R. Clinical experiences associated with anaesthesia training. Ann Roy Coll 1993; 26: 363-7.

12 Cheney $F W$. Anesthesia patient. Safety and professional liability continues to improve. ASA newsletter 1997; $61(6)$. 